\title{
Pioneer in Biomedical Optics: Introduction to the Special Section in Honor of Steven L. Jacques
}

\author{
Andreas H. Hielscher \\ Jessica Ramella-Roman \\ Lihong V. Wang
}




\title{
Pioneer in Biomedical Optics: Introduction to the Special Section in Honor of Steven L. Jacques
}

\author{
Andreas H. Hielscher \\ Columbia University \\ Department of Biomedical Engineering \\ New York, New York 10027 \\ United States \\ Jessica Ramella-Roman \\ Florida International University \\ Department of Biomedical Engineering and Herbert Wertheim College of Medicine \\ Miami, Florida 33174 \\ United States \\ Lihong V. Wang \\ California Institute of Technology \\ Cherng Department of Medical Engineering \\ Pasadena, California 91125 \\ United States
}

We present this special section of the Journal of Biomedical Optics in honor of Steven L. Jacques, $\mathrm{PhD}$, a leading pioneer in the field of biomedical optics. In the late eighties, Steve was part of a small group of researchers from various disciplines who clearly saw the potential of light and optics for new approaches to medical diagnostics and treatment of various diseases. Since then the field has grown exponentially and biomedical optics has become an integral part in many medical disciplines. Through his work and many seminal contributions, Steve has actively shaped this development.

Steve followed an unconventional path, receiving a BS in biology at MIT, and an MS in electrical engineering and computer science and a $\mathrm{PhD}$ in biophysics and medical physics from the University of California-Berkeley (1984). Already at this early stage, his interest in finding novel ways to characterize human tissue was evident. In 1979, he published his very first paper, "Linear measurement of the water content of the stratum corneum of human skin using a microwave probe," in the Journal of Sound and Vibration. ${ }^{1}$ He would go on to explore this topic in greater detail during his $\mathrm{PhD}$ research. After receiving his doctoral degree, he moved back east and started his postdoctoral work at the Wellman Center for Photomedicine at Massachusetts General Hospital under the guidance of the well-known dermatologist John Albert Parrish, MD. Dr. Parrish, who founded the Wellman Center, was one of the very early pioneers in the field of photochemotherapy, using it to treat skin disorders such as psoriasis. In his lab, Steve was first introduced to the idea of using light for medical applications. He initially focused on the use of lasers for fragmentation of urinary calculi and percutaneous drug delivery. Steve soon realized that, to be quantitative about these applications, he needed a mathematical tool to calculate light propagation in tissue and accurately determine optical properties of tissues. Together with Scott Prahl, he wrote his first true blockbuster paper, "Modeling optical and thermal distributions in tissue during laser irradiation,"

(C) 2018 Society of Photo-Optical Instrumentation Engineers (SPIE) which has now been cited over 300 times. In that paper, he introduced the diffusion approximation solution to the one-dimensional optical transport equation and used it to interpret data obtained from tissue slices placed between two integrating spheres. This approach would soon become, and still is today, a standard method to determine optical properties of biomedical tissues. This work was followed by another modeling paper from his team, "Light distributions in artery tissue: Monte Carlo simulations for finite-diameter laser beams." It was the first time that Monte Carlo methods had been used to describe light propagation in tissue.

Apparently realizing that the era of quantitative biomedical optics had arrived, Dr. Andrew von Eschenbach, MD, who would later go on to become the director of the National Cancer Institute $(\mathrm{NCl})$ at the National Institutes of Health (NIH), recruited Steve to the world-renowned MD Anderson Cancer Center. As an assistant professor of urology/biophysics he then led the Laser Biology Laboratory and landed

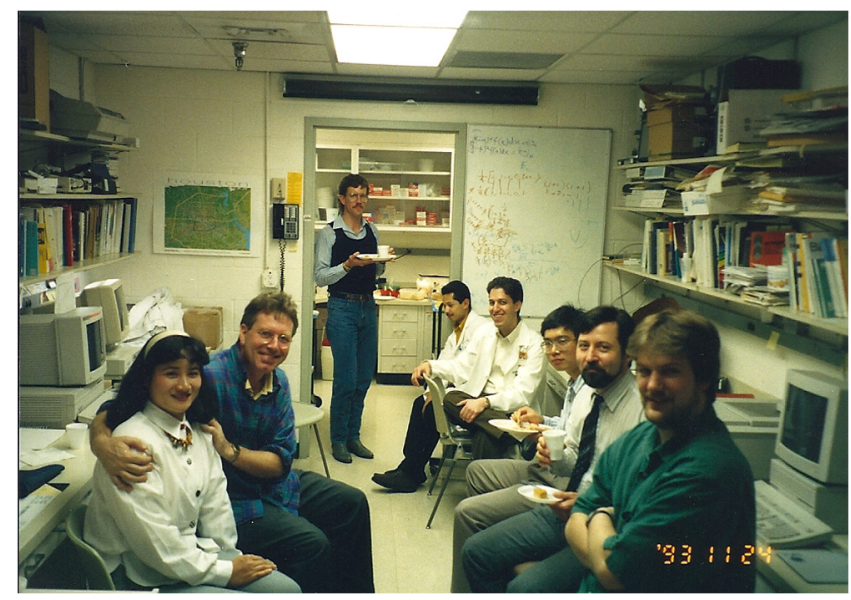

Steve and his team at MD Anderson Cancer Center, Houston, Texas, in 1993, including Andreas Hielscher, Alexander Oraevski, and Lihong Wang, on the right. 


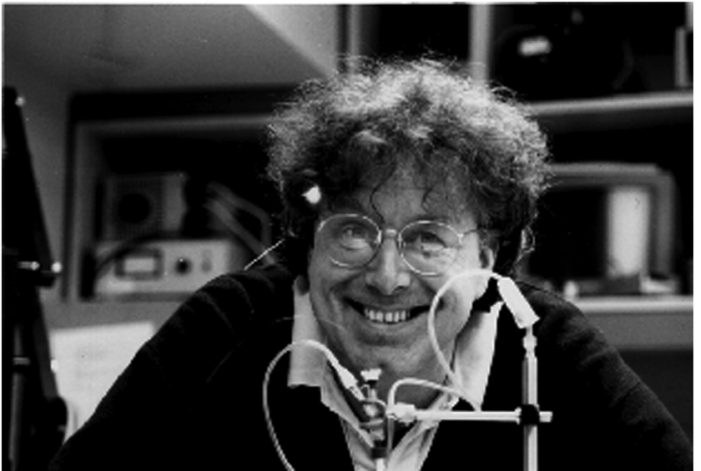

Steve at Oregon Health and Science University in Portland (circa 2002).

his first NIH grant, entitled "Dosimetry of laser/tissue interactions." As part of this effort, Steve continued his work on Monte Carlo methods and together with his postdoc, Lihong Wang, he went on to develop the now famous MCML Monte Carlo simulation package. They made this package publicly available along with the source code, and today this tool is the de facto gold standard in the field. It has been widely used worldwide to simulate photon propagation in biological tissues for both teaching and research purposes. With over 3,000 citations, the related publication ${ }^{4}$ is one of the most often cited papers in our field. The companion paper ${ }^{5}$ has been cited hundreds of times as well. This code and derivations of it have become ubiquitously used throughout the biomedical optics world; the creation and open source distribution of it was the impetus for much of the growth in understanding of how light-tissue interactions happen.

Still in Texas, Steve's team also developed a novel noninvasive technology for the determination of bilirubin concentration in the skin.,7 This system was further developed into a hand-held spectrometer and was applied to noninvasively measure hyperbilirubinemia (jaundice) in newborns. Patented, licensed, and FDA approved, this device is currently distributed by Phillips under the name BiliCheck. It replaces heel stick tests and is now commonly used in clinics

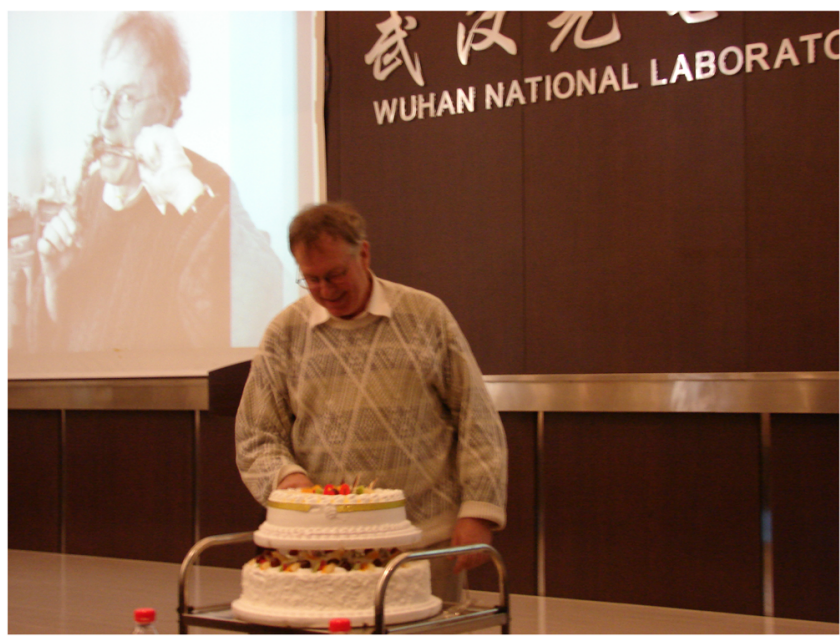

Steve's 60th birthday party held at the Chance Center, Huazhong University of Science and Technology, Wuhan, China

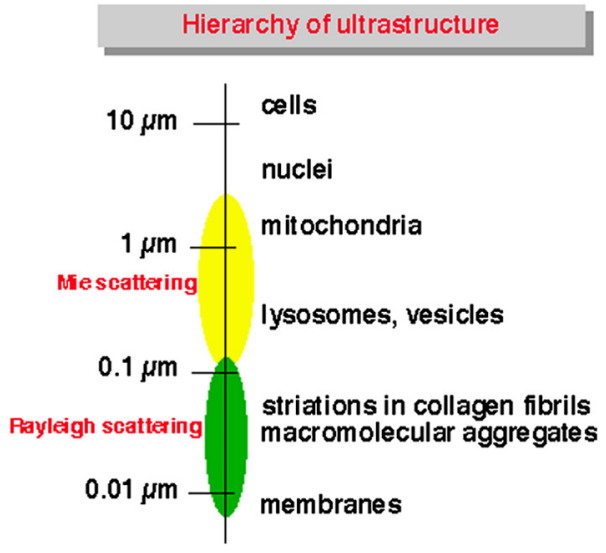

Hierarchy of ultrastructure in scattering, an image from omlc.ogi.edu, which has been reproduced countless times.

for neonatal care. As of 2018, over 230 million newborns have been tested with the device.

In 1996, Steve joined the Oregon Health and Science University in Portland as professor of dermatology and biomedical engineering. There he joined the newly founded Oregon Medical Laser Center, where he worked on a number of novel applications of light tissue interaction, developing novel uses of optical technologies for both therapy and diagnosis. He studied how polarized light travels in biological media and developed some of the first imaging systems for dermatology applications including a hand-held polarized light camera to visualize skin cancer margins and guide surgical excision. He also developed in vivo subnanometer measurements of vibration of the cochlear membrane of the inner ear in animal models and explored the effect of short ablation arising from stress-induced cavitation. Furthermore, he curated the omlc.ogi.edu website with Scott Prahl, at that time one of the very few divulgatory sites in biophotonics. The website contained a very popular spectral database for biological tissue, as well as a series of lessons introducing biophotonics to the general public. Steve is known as a living library of tissue optical properties. His review on the topic ${ }^{8}$ has been cited over

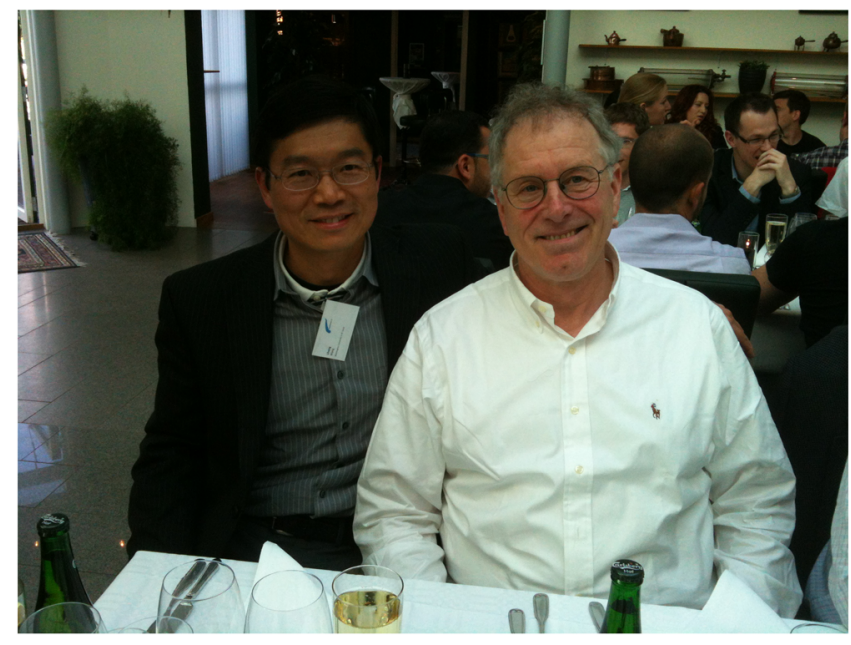

Steve Jacques and Lihong Wang at the 2013 Biophotonics Summer School, Ven, Sweden. 


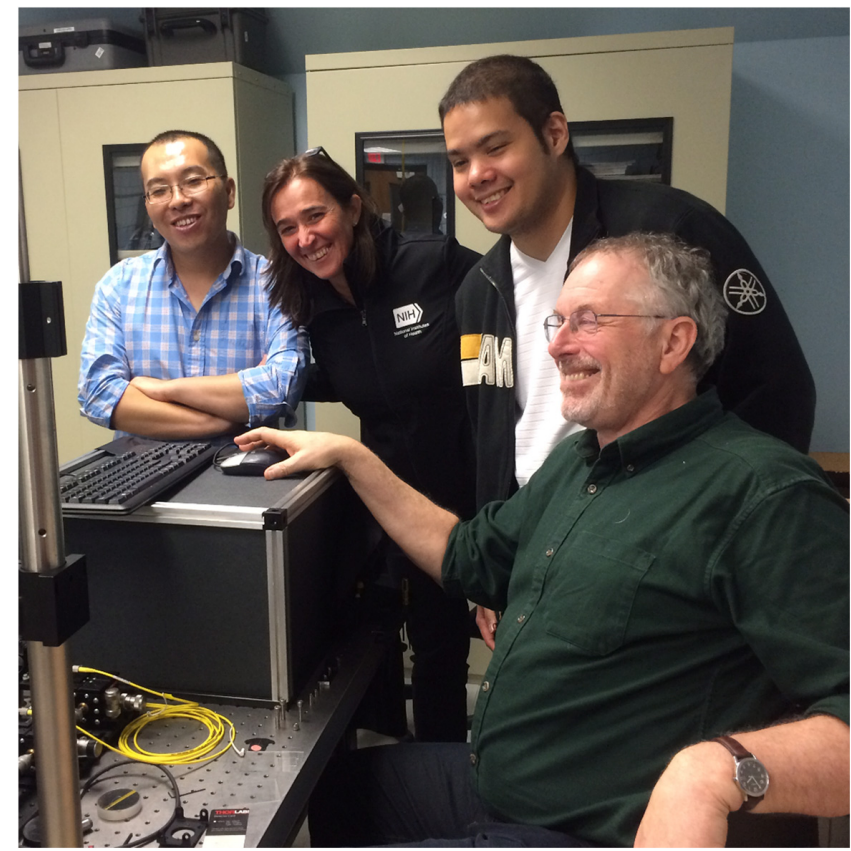

Steve mentoring students of all ages in Miami (2016).

1,000 times. Steve is also a frequent contributor to the Journal of Biomedical Optics. For example, his publication on polarized light imaging of the skin is among the most cited papers in the journal. ${ }^{9}$

Steve's diverse training in biology, electrical engineering and physics has been instrumental in his approach to scientific discovery; many of his insights come from his great understanding of the interplay between these disciplines; it has also proven to be an asset when disseminating his many scientific ideas. Over the years Steve has demonstrated a passion for teaching and a remarkable dedication to mentoring new generations of investigators. He has been a popular instructor at the prestigious biennial International Graduate Summer School in Biophotonics, held on the Island of Ven, Sweden, since its inception in 2003, and a regular participant in many other tutorials or summer programs in biomedical optics or biophotonics. He regularly visited the Britton Chance Center for Biomedical Photonics at Huazhong University of Science and Technology, Wuhan, China, and taught summer courses on biomedical optics. When the Chance Center hosted Steve's 60th birthday party in 2010, Lihong pleasantly discovered that the acronym for their Monte Carlo package, MCML, happened to match Steve's birth year in Roman numerals. At SPIE's Photonics West symposium, Steve has been teaching the short course on tissue optics since the early 1990s. His tutorial on diffuse light transport has been widely read and cited. ${ }^{10}$ As Steve's former mentees, all three of us have been inspired by his research and benefited tremendously from his training.

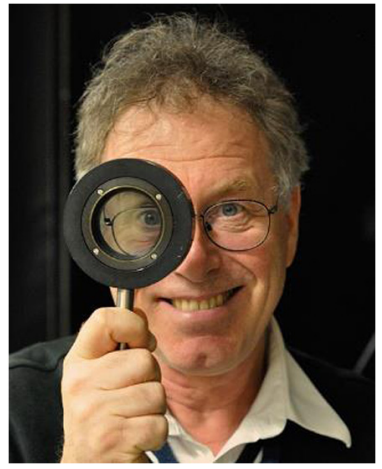

Steve Jacques, Professor of the Practice of Biomedical Engineering, Tufts University.

Recently, Steve has started a new adventure moving back to the Boston area as a professor at Tufts University. He continues his work in developing novel microscopes that are sensitive to the ultrastructure of cells and tissues, as well as teaching biophotonics to a new generation of graduate and undergraduate students. His infectious curiosity and zest for discovery come out in every interaction he has with students in the lab or in the classroom. As a cofounder, pioneer, and mentor of many leaders in one of the most innovative new fields in medicine, Steve has richly earned the honor of this special section.

\section{References}

1. S. L. Jacques, "Linear measurement of the water content of the stratum corneum of human skin using a microwave probe," J. Sound Vib. pp. 180-182 (1979).

2. S. L. Jacques and S. A. Prahl, "Modeling optical and thermal distributions in tissue during laser irradiation," Laser. Surg. Med. 6, 494-503 (1987).

3. M. Keijzer et al., "Light distributions in artery tissue: Monte Carlo simulations for finite-diameter laser beams," Laser. Surg. Med. 9(2), 148-154 (1989).

4. L. V. Wang, S. L. Jacques, and L. Zheng, "MCML-Monte Carlo modeling of light transport in multi-layered tissues," Comput. Method Programs Biomed. 47(2), 131-146 (1995).

5. L. V. Wang, S. L. Jacques, and L. Zheng, "CONV-convolution for responses to a finite diameter photon beam incident on multi-layered tissues," Comput. Meth. Programs Biomed. 54(3), 141-150 (1997).

6. S. L. Jacques, D. Oelberg, and I. Saidi, "Method and apparatus for optical measurement of bilirubin in tissue," U.S. Patent No. 5,353,790 (1994).

7. S. L. Jacques et al., "Developing an optical fiber reflectance spectrometer to monitor bilirubinemia in neonates," Proc. SPIE 2975, 115-124 (1997).

8. S. L. Jacques, "Optical properties of biological tissues: a review," Phys. Med. Biol. 58(11), R37 (2013).

9. S. L. Jacques, J. C. Ramella-Roman, and K. Lee, "Imaging skin pathology with polarized light," J. Biomed. Opt. 7(3), 329-341 (2002).

10. S. L. Jacques and B. W. Pogue, "Tutorial on diffuse light transport," J. Biomed. Opt. 13(4), 041302 (2008). 